\title{
SIRS Model of Passengers' Panic Propagation under Self-Organization Circumstance in the Subway Emergency
}

\author{
Haifeng Zhao, Jie Jiang, Rongyu Xu, and Yang Ye \\ School of Economic and Management, Tongji University, Shanghai 200092, China \\ Correspondence should be addressed to Haifeng Zhao; hfzhao@tongji.edu.cn
}

Received 31 January 2014; Revised 13 April 2014; Accepted 14 April 2014; Published 8 May 2014

Academic Editor: X. Zhang

Copyright ( 2014 Haifeng Zhao et al. This is an open access article distributed under the Creative Commons Attribution License, which permits unrestricted use, distribution, and reproduction in any medium, provided the original work is properly cited.

\begin{abstract}
Subway emergency may lead to passengers' panic, especially under self-organizing circumstance, which will spread rapidly and have an adverse impact on the society. This paper builds an improved SIRS model of passengers' panic spread in subway emergency with consideration of passengers' density, the characteristic of subway car with the confined space, and passengers' psychological factors. The spread of passengers' panic is simulated by use of Matlab, which draws the rules of how group panic spreads dynamically. The trend of stable point of the infection ratio is analyzed by changing different parameters, which help to draw a conclusion that immunization rate, spontaneous immune loss rate, and passenger number have a great influence on the final infected ratio. Finally, we propose an integrated control strategy and find the peak of passengers' panic and the final infected ratio is greatly improved through the numerical simulation. The research plays a vital role in helping the government and subway administration to master the panic spread mechanism and reduce the panic spread by improving measures and also provides certain reference significance for rail system construction, emergency contingency plans, and the construction and implementation of emergency response system.
\end{abstract}

\section{Introduction}

Since the first subway, named LMS (London metropolitan subway), was built in 1863, nowadays, there are more than $6000 \mathrm{kms}$ lines in more than 100 cities of 35 countries and regions in the world that have been constructed. With the growing population and the increasing speed of urbanization, the subway is becoming the first choice for the public transportation of passengers to travel due to the advantage of the large volume, fast speed, convenience, and so forth. However, because of the large population density and the small enclosed space of the metro, the panic caused by emergency would be spread rapidly and result in the confusion, which would further magnify the impact of the entire event. In 2003, up to 25 million passengers were trapped in the subway because of a major blackout in London and caused panic, leading to great dissatisfaction. What is more, the spreading panic of passengers may cause a number of secondary accidents. For example, in the evening of September 2, 2013, Guangzhou Metro Line 2 subway suddenly braked and another one in the back caught up, the passengers in the back of carriage mistook it as a rearend accident and fled toward the front of the cars with shouting, and some passengers were injured in the stampede. At noon of March 4, 2014, two youngsters played antiwolf spray in the back of Guangzhou Metro Line 5 and suddenly pungent odor was emitted, panicking passengers rushed to the front of the cars continuously and caused stampede, wounds, and luggage scattering, seriously disrupting social order and public transport. Even more, those incidents will further lead to people's psychological panic and it will not only affect the individual physical and mental health but also cause serious damage to the politics, economy, and social life. Therefore, the study of passengers' psychology and emotion in the emergency has got extensively concerned.

The related research on subway emergencies began in the 1990s, and the majority concentrated in the emergency was evacuation capability assessment $[1,2]$, emergency evacuation strategies [3], emergency location [4], and establishing emergency system $[5,6]$. In addition, some scholars have established emergency evacuation models [7-10] to simulate subway emergencies scenarios. But most of these studies only 
considered the safety of passenger; few scholars considered the psychological impact of the passengers caused by the subway emergencies. When subway emergencies occur, the normal social environment will be disrupted and inner tension will be expanded, and when the psychological tension reaches a certain level, it will cause group psychological panic, the inherent performance, which is panic emotion. Earlier studies suggested that people would lose essential humanity and fall into fear beast in the face of terrible disaster. Quarantelli [11] believed that panic is a collection of selfish behavior; when psychological panic occurs, people are more concerned with their own destiny rather than collective one. Le Bon [12] thought that people are impulsive and irrational and lack accountability, due to the factors such as anonymity, infection, and hint. The individual will lose rationality and responsibility, once he entered the masses, and then shows impulsive, brutal antisocial behavior. There are some researches on factors for panic; Mawson [13] pointed out that the panic comes from awareness and has relationship with social organization, culture, environment, situational factors, and social control. Aguirre [14] pointed out that the generation of panic is influenced by architecture structure, group members, group density, the relationship between the groups, the resource situation, and the amount of information. Panic can be described as the psychological panic as well as the panic behavior. Panic in behavioral performance is panic behavior. On the study of panic behavior, Kelley et al. [15] provided the simulation study about cluster behavior under the panic environment. Ebihara et al. [16] explored the behavior of individual panic. Saloma et al. [17] considered the existence of the self-organization queue behavior and freedom scale behavior. Low [18] assumed that groups are made up of different individuals with ideas and the ability, establishing a quantitative model to study the characteristics of irrational group behavior. He thought those group behaviors are generated because the widespread impact occurred under the situation of relatively spontaneous behavior and disorganized situation and it is dependent on stimulation of each participant. Helbing et al. [19] studied simulation dynamic characteristic of the panic to escape. As a special group behavior, the fugitive groups behavior in emergencies shows imitation, no purpose, spontaneity, vulnerability, and other nine characteristics. Because panic is reflected by the panic behavior, therefore, we can conclude that panic can be infectious.

Two of the most common and far-reaching models, SIS model [20] and SIR model [21], which were originally used for propagation mechanism of the virus, could be used and thoroughly researched for studying the infection and communication processes of crisis information [20-33]. Pastor-Satorras et al. [34-37] classified the complex nodes in the network according to their value and established the SIS model. Moreno et al. [38, 39] confirmed that there exist a certain number of infected nodes in the end, even if the initial infection is very low by applying the SIR model. Li et al. [40] believed that, in real life, there are some viruses that cannot be immune for all life and built a complex heterogeneous network SIRS epidemic model to a more realistic portrayal of the spread of infection. Based on this work, Zhao et al. [24, 26, 39] applied the epidemic model to the study of spread of rumors issues. Yuanyuan et al. [41] applied SIR epidemic model to the stock market crisis communication research. Scholars have also studied the problem for the specific context and the classic epidemic model has been improved. In the study of the propagation of the disease, taking into account the nonuniform interaction between nodes, Dybiec [23] extended the classical SIR model. Sekiguchi et al. [40, 42, 43] studied the distributed delay characteristics of infectious diseases in the model. Tchuenche et al. [44-47] believed that the total population is changing in real life due to the birth and death rates. Li et al. [48-51] thought that the infection rate and cure rate in the spread of disease are nonlinear. Zhao et al. [24] used infectious disease model to study the rumor spread issue. Considering the characteristics of rumors spread and social networks, they added forgetting factor in the model to describe the node spontaneous autoimmune conditions and then concluded that forgetting rate coefficient and immunization rates have a significant impact on the spread of rumors in the social network. Between the listed companies and the main stock holders, Yuanyuan et al. [41] established a susceptible-infected-removed model of crisis spreading (SIR) in the stock markets by taking the mutual influences into account which resulted from reduced cash flows or the fracture of capital chain. Then, a numerical simulation is used to analyze the crisis spreading in the correlated networks when the networks meet the random failure or the intentional attacks. These models did not consider the crowd density, dynamic infection rates, and some other conditions, whereas these conditions are very important in the study of the panic spreading in subway emergencies. This paper simulates the panic spread of the passengers under the subway emergency based on the epidemic models.

In summary, with the occurrence of unexpected events, people's ability to think will draw down. It will be more likely for them to accept the implied information, and they will be much more thirsty for information than usual. At this time, people's psychological emotions are in extreme tension and become panicked, and the most outstanding performance is the herd mentality of the individual. When some of the passengers appear confused in verbal expression, actions, or abnormal panic expression, panic will infect neighboring passengers. Then, the neighboring passengers will probably become panicked and then spread panic mood in the whole metro. When the emergency calms down, part of passengers may calm down, and then individuals become immune with a certain probability. However, immunity is not permanent; if their surroundings are still in a state of panic due to their own poor mentality, the group will become susceptible with certain probability, which makes it very similar to the propagation mechanism of SIRS model to a certain extent. Therefore, we chose SIRS model as an analysis model to study the spread of panic in the subway emergency. It is worth noting that, comparing with the classical SIRS model which describe the spread of viruses or rumors, the SIRS model describing the passengers also exist three corresponding state that are healthy state $(S)$, infection status $(I)$ and immune status $(R)$, but this one is established based on the actual existence of exchanges 
between the panic passengers in the subway. First of all, we need to verify whether it is peak period. Secondly, panic may spread from one of the cars to another in the narrow closed space, and therefore the probability to be infected or lose immunity between the passengers is not fixed. But the infection rate and the immunity loss rate are nonlinear functions rather than constants in the situation that panic spreads, and the immunization rate is related to the state of the emergency situation. Thirdly, the infected passengers are likely to be spontaneously immune because of their own mentality, and immune passengers are likely to spontaneously lose immunity and become susceptible groups due to the psychological factors of passengers. Besides, it should be emphasized that we only studied the self-organizing behavior without considering the participation of the government and the media.

It is important to grasp the mechanism of panic spread and improving measures to reduce the spread of panic, which will provide a reference for providing the rail system emergency plans and emergency information system construction and implementation. In this research, the study method on virus spreading is introduced to the subway emergencies to analyze the psychological and behavioral research, and then the SIRS model of emotional panic spread of subway passengers in emergencies is built, and the spreading process is analyzed by quantitative analysis and numerical simulation to reveal its spread rule and predict its spreading trends. The main contents are as follows. In Section 2, the subway passengers in the specific context in subway emergencies are classified; model assumptions are provided; then the model with improved parameters is established. Section 3 analyzes the model stability and Section 4 gives the simulation of the model. Finally, in Section 5, the findings of this study are summarized and the direction of future research is pointed out.

\section{The Propagation Model of Panic in the Subway Emergency}

2.1. The Definition of the Passenger State Node. In the event of the emergency, passengers in the cars may be in three kinds of states, $S$ state, $I$ state, and $R$ state.

Susceptible state ( $S$ state) is as follows: the susceptible ones are comprised of individuals who are not in panic and are susceptible to become panicked, who are also called susceptible persons. The ratio of susceptible person is $S(t)$, indicating the ratio of passengers that have not been infected and remain calm at the time $t$.

Infection state ( $I$ state) is as follows: the infected ones are those who are in panic and spread the panic to others; panic can be caused by the emergency itself or the fact that they are infected by the surroundings. And the ratio of infected ones is $I(t)$, indicating the ratio of passengers that have become panicked with the ability to spread panic at the time $t$.

Immune state ( $R$ state) is as follows: the passengers who were affected by panic but later become patient and not afraid in the eased situation are known as the immune ones. And the ratio of immune ones is $R(t)$, indicating the ratio of

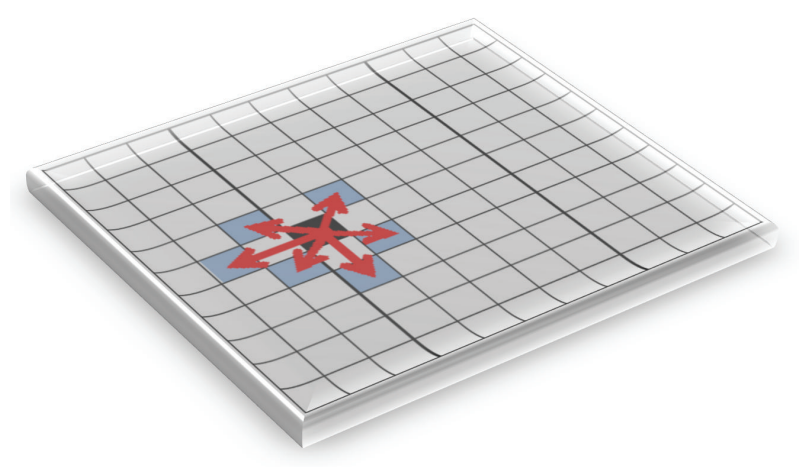

FIgURE 1: The individual contact model in dimensional lattice diagram.

passengers who are in immune state from the infection state at the time $t$. However, these passengers are also likely to become susceptible once again.

\subsection{The Model Assumptions}

(1) The total passenger number in the subway is always maintained at a constant $N$.

(2) The passengers in the subway cars are uniformly distributed and the average degree of mutual contact between individuals is $\langle k\rangle$ during the normal driving which means at the off-peak or off-peak time.

(3) There are no birth or death issues in the process of panic spreading.

(4) Suppose that the probability of a susceptible passenger being infected by an infected one is constant, so the probability of an immune person losing immunity after contact with an infected one.

(5) At the initial period of the emergency, there are only the passengers who are in susceptible state and infection state but no immune state.

\subsection{Improved Model Parameters}

2.3.1. Population Density. The variable of passenger density is $\rho$, which may vary from time to time; the moving crowd can be simulated by using the two-dimensional regular lattices; we assume that the metro is an area of $L * W$ zone, where $L$ is the length of the metro and $W$ is the width of it. In the subway, the total number of this passenger group is $N$ and the group makes random motion in the two-dimensional lattice. In the graph, each square represents an individual. The state to which he belongs is not taken into account and it just shows the mutual contact between the individual in the middle and the surrounding one in the graph. Besides, the number of the contacted individuals is $\langle k\rangle$, which equals 6 , and it is the degree to the node. The simulated passenger contact model is shown in Figure 1. 


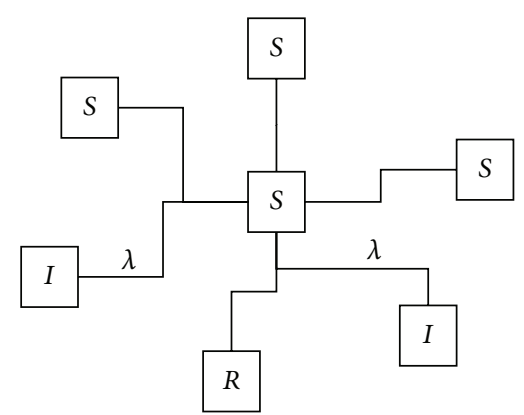

FIGURE 2: The contact rendering in unit space.

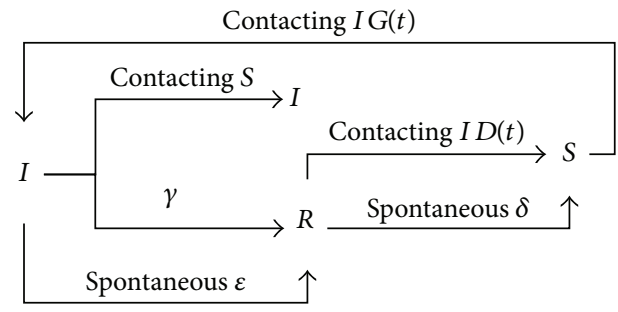

FIGURE 3: Structure of spreading process.

Therefore, the passenger density can be expressed as follows:

$$
\rho=\frac{N}{L * W} .
$$

2.3.2. The Infection Rate. As is known about the assumption about the same infection rate among individuals and the constant probability $\lambda$ to converse to the panic state when the healthy person contacts the infected person and susceptible people contact an infected person, the probability of susceptible individuals infected is related to the number of infected individuals around the susceptible passengers. As the susceptible individual degree is $\langle k\rangle, \rho\langle k\rangle I(t)$ means the number of infected state individuals whom the susceptible individuals may contact at time $t ;(1-\lambda)$ represents the no-infection probability after the healthy person contacts an infected person, and the number of the infections around the healthy is $\rho\langle k\rangle I(t)$, so the no-infection probability after the healthy person contacts all the infected persons around is $(1-\lambda)^{\rho\langle k\rangle I(t)}$ and the probability of being infected is $1-(1-\lambda)^{\rho\langle k\rangle I(t)}$; the contact rendering in unit space is shown in Figure 2.

As a result, we can get the infection rate of the susceptible individuals, which is as follows:

$$
G(t)=\left[1-(1-\lambda)^{\rho\langle k\rangle I(t)}\right] .
$$

2.3.3. The Immunization Rate. Immune rate represents the recovery ability of the infected individual. As we only study self-organization without considering the impact on passengers panic by another organization, the immunization rate in the model is related to the calm-down speed and the dramatic degree of the emergency. As some emergencies are caused by the rumor from some passengers in the subway, so it can become immune state when finally the scared passengers discover the truth of events; we assume that this kind of immunization rate is $\gamma$. Besides, the immunization rate is related to the psychological state, educational level, age, and so forth, and the panic passengers can spontaneously become immune with the spontaneous immune probability of $\varepsilon$.

2.3.4. The Immune Loss Rate. Based on the assumption (4) in Section 2.2, the immune losing rate among individuals is the same when the immune contact with the infected person, we assume that this kind of immune loss rate is $\beta$. The probability of immune individual becoming susceptible is related to the number of infected individuals around. So the probability function of the immune loss rate of immune individuals is

$$
D(t)=\left[1-(1-\beta)^{\rho\langle k\rangle I(t)}\right] .
$$

At the same time, even when there is noninfected individual, there are still a handful of individuals becoming susceptible to the factors such as the psychological mentality, marking this state of immunization rate as spontaneous immunization rate $\delta$.

2.3.5. The Model. This kind of spreading process is shown in Figure 3.

Based on the assumption and conditions, we established a subway emergencies propagation model which improves SIRS epidemic model, and it is shown as follows:

$$
\begin{gathered}
\frac{d S(t)}{d t}=-\left[1-(1-\lambda)^{\rho\langle k\rangle I(t)}\right] S(t) \\
+\left[1-(1-\beta)^{\rho\langle k\rangle I(t)}\right] R(t)+\delta R(t) \\
\frac{d I(t)}{d t}=\left[1-(1-\lambda)^{\rho\langle k\rangle I(t)}\right] S(t)-(\gamma+\varepsilon) I(t) \\
\frac{d R(t)}{d t}=(\gamma+\varepsilon) I(t)-\left[1-(1-\beta)^{\rho\langle k\rangle I(t)}\right] R(t)-\delta R(t) .
\end{gathered}
$$

\section{The Lyapunov Stability for the New Epidemic Model}

The model represented above is established on the assumption that the population size $N$ is a constant. The condition $S(t)+I(t)+R(t)=1$ can omit the equation of $R(t)$ by $S(t)$ and $R(t)$, so the two-dimensional system is given by

$$
\begin{aligned}
\frac{d S(t)}{d t}=- & {\left[1-(1-\lambda)^{\rho\langle k\rangle(t)}\right] S(t) } \\
+ & {\left[1-(1-\beta)^{\rho\langle k\rangle I(t)}+\delta\right](1-S(t)-I(t)) } \\
\frac{d I(t)}{d t}= & {\left[1-(1-\lambda)^{\rho\langle k\rangle I(t)}\right] S(t)-\gamma I(t) . }
\end{aligned}
$$


Let $d S(t) / d t=0$ and $d I(t) / d t=0$; then

$$
\begin{aligned}
& -\left[1-(1-\lambda)^{\rho\langle k\rangle I(t)}\right] S(t) \\
& \quad+\left[1-(1-\beta)^{\rho\langle k\rangle I(t)}+\delta\right](1-S(t)-I(t))=0 \\
& {\left[1-(1-\lambda)^{\rho\langle k\rangle I(t)}\right] S(t)-(\gamma+\varepsilon) I(t)=0 .}
\end{aligned}
$$

Theorem 1. When $-\langle k\rangle \rho \ln (1-\lambda) /(\gamma+\varepsilon)<1$, there is one and only disease-free equilibrium for Model (4) in the positive invariant set, and the equilibrium is $S(t), I(t)=(1,0)$.

Proof. The Jacobian matrix $J$ is as follows:

$$
J=\left(\begin{array}{cc}
-\delta, & \langle k\rangle \rho \ln (1-\lambda) \\
0, & -(\gamma+\varepsilon)-\langle k\rangle \rho \ln (1-\lambda)
\end{array}\right) .
$$

When $|J|>0$, it means the trace of matrix $\operatorname{tr}(J)=-(\gamma+$ $\varepsilon)-\langle k\rangle \rho \ln (1-\lambda)<0$, and it also means when $-\langle k\rangle \rho \ln (1-$ $\lambda) /(\gamma+\varepsilon)<1,(s(t), I(t))=(1,0)$ is stable.

Theorem 2. When $1<-\langle k\rangle \rho \ln (1-\lambda) /(\gamma+\varepsilon)<1 / S^{*}$, there exists the local asymptotically stable equilibrium point $\left(S^{*}, I^{*}\right)$.

Assuming that $\left(S^{*}, I^{*}\right) \neq(1,0)$ is an another positive equilibrium state of system (6), the objective is to prove if there exist one pair or more pairs $\left(S^{*}, I^{*}\right)$ to ensure the existence of the solution when $-\langle k\rangle \rho \ln (1-\lambda) /(\gamma+\varepsilon)>1$.

Let $G(I)=\left[1-(1-\lambda)^{\rho\langle k\rangle I(t)}\right]$ and $D(I)=[1-$ $\left.(1-\beta)^{\rho\langle k\rangle I(t)}\right]$ for calculating easily. So (6) can be shown as

$$
\begin{gathered}
-G(I) S+(D(I)+\delta)(1-S-I)=0 \\
G(I) S-(\gamma+\varepsilon) I=0 .
\end{gathered}
$$

Substituting the point $\left(S^{*}, I^{*}\right)$ in system (8), we can rewrite this system as

$$
\begin{gathered}
-G\left(I^{*}\right) S^{*}+\left(D\left(I^{*}\right)+\delta\right)\left(1-S^{*}-I^{*}\right)=0 \\
G\left(I^{*}\right) S^{*}-(\gamma+\varepsilon) I^{*}=0 .
\end{gathered}
$$

We use the elimination method as

$$
-(\gamma+\varepsilon) I^{*}+\left(D\left(I^{*}\right)+\delta\right)\left(1-\frac{(\gamma+\varepsilon) I^{*}}{G\left(I^{*}\right)}-I^{*}\right)=0
$$

Let $H(I)=-(\gamma+\varepsilon) I+(D(I)+\delta)(1-((\gamma+\varepsilon) I / G(I))-I)$, where $f(0)=g(0)=0, f^{\prime}(0)>0, g^{\prime}(0)>0, f^{\prime \prime}(0)<0$, and $g^{\prime \prime}(0)<0$; then

$$
\begin{gathered}
H(0)=\delta\left(1-\frac{\gamma+\varepsilon}{G^{\prime}(0)}\right)>0 \\
H(1)=-(\gamma+\varepsilon)-\frac{\gamma+\varepsilon}{G(1)}(D(1)+\delta)<0
\end{gathered}
$$

$H^{\prime}(I)$

$$
\begin{aligned}
= & -(\gamma+\varepsilon)+D^{\prime}(I)\left(1-\frac{(\gamma+\varepsilon) I}{G(I)}-I\right) \\
& +(D(I)+\delta)\left(1-I-\frac{(\gamma+\varepsilon)\left[G(I)-I G^{\prime}(I)\right]}{G^{2}(I)}\right)<0 .
\end{aligned}
$$

So a point makes the system stable exist, and the point is not equal to zero. The Jacobian matrix of (8) is

$$
J=\left(\begin{array}{cc}
-G\left(I^{*}\right)-D\left(I^{*}\right)-\delta & -G^{\prime}\left(I^{*}\right) S^{*}-D^{\prime}\left(I^{*}\right)\left(1-S^{*}-I^{*}\right)-D\left(I^{*}\right)-\delta \\
G\left(I^{*}\right) & G^{\prime}\left(I^{*}\right) S^{*}-(\gamma+\varepsilon)
\end{array}\right) .
$$

When the trace of matrix $G\left(I^{*}\right)+D\left(I^{*}\right)+\delta-G^{\prime}\left(I^{*}\right) S^{*}+$ $\gamma+\varepsilon>0$, it can be simplified as $G^{\prime}\left(I^{*}\right) S^{*}<\gamma+\varepsilon$.

$G^{\prime}(I)$ is decreasing, and $G^{\prime}(I)>0$, so if it satisfied the condition $G^{\prime}(0) S^{*}<\gamma+\varepsilon$, that is, $-\langle k\rangle \rho \ln (1-\lambda) /(\gamma+\varepsilon)<$ $1 / S^{*}$, the system (6) can achieve a globally asymptotically stable, and, at this time, the infected people will not gone, but the proportion of it can asymptotically stable as a constant $\left(S^{*}, I^{*}\right)$.

\section{Numerical Simulation}

4.1. Dynamic Simulation of Panic Spreading. After actual survey, we make numerical simulation of the panic spread model built in Section 2 and set model parameters as number of passengers $N=1400$, length and width of the subway car $L=164.78$ meters and $W=3$ meters, the average degree of mutual contact between individuals $\langle k\rangle=6$, and initial proportion of the susceptible and the infected
$S(t)=0.95, I(t)=0.05$. Given the confined space in the subway car, panic will spread quickly once emergency happens, so we set the infected rate a higher value $\lambda=0.9$. As the immunization rate is related to the development, $\gamma$ is set to be 0.4 . The immune loss rate is the rate of passengers who become susceptible again, influenced by the infected passengers around, so we set $\beta=0.1$. Meanwhile, the probability of the infected persons turning to be immune by self-mentality is generally higher than that of the immune persons turning to be susceptible, and we set $\varepsilon=0.1$ and $\delta=0.05$. By solving the model equations using the ODE45 arithmetic of Matlab, we change the proportion of the susceptible, the infected, and the recovered persons in the spread process of panic, as shown in Figures 4(a) and 4(b).

According Figure 4(b), we can find out that, with the rapid spread of panic in the subway car, the proportion of the infected persons increases quickly from the initial 5\% to the maximum value $52.14 \%$ with 730 infected passengers 

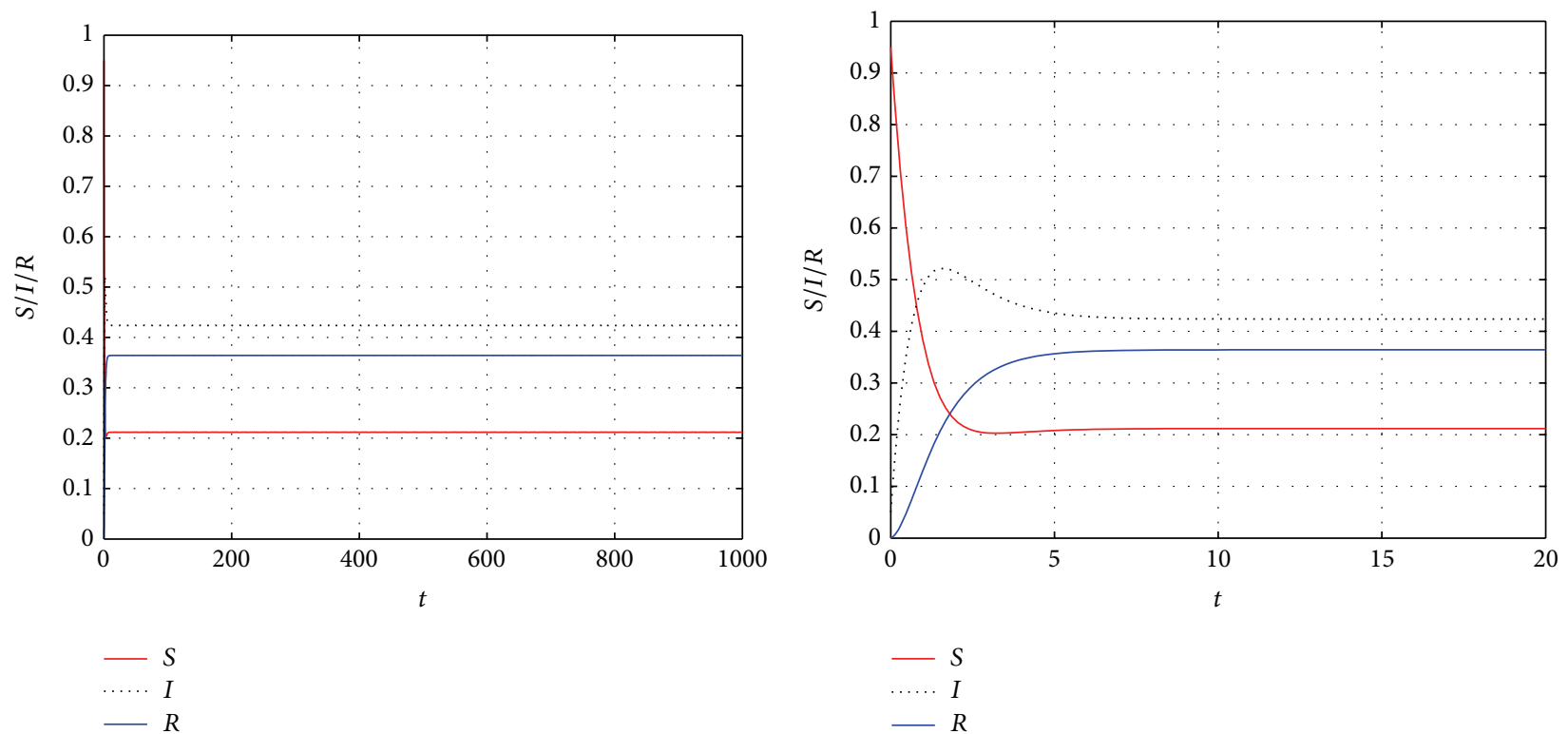

(a) Extended figure of (b)

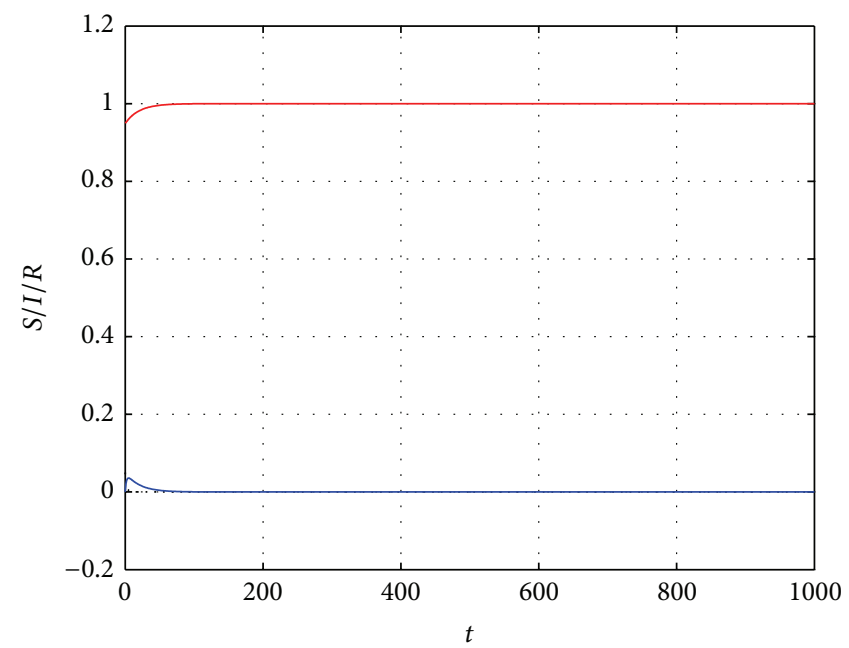

(b) $\lambda=0.9, \gamma=0.4, \beta=0.1, \varepsilon=0.1, \delta=0.05$, and $N=1400$

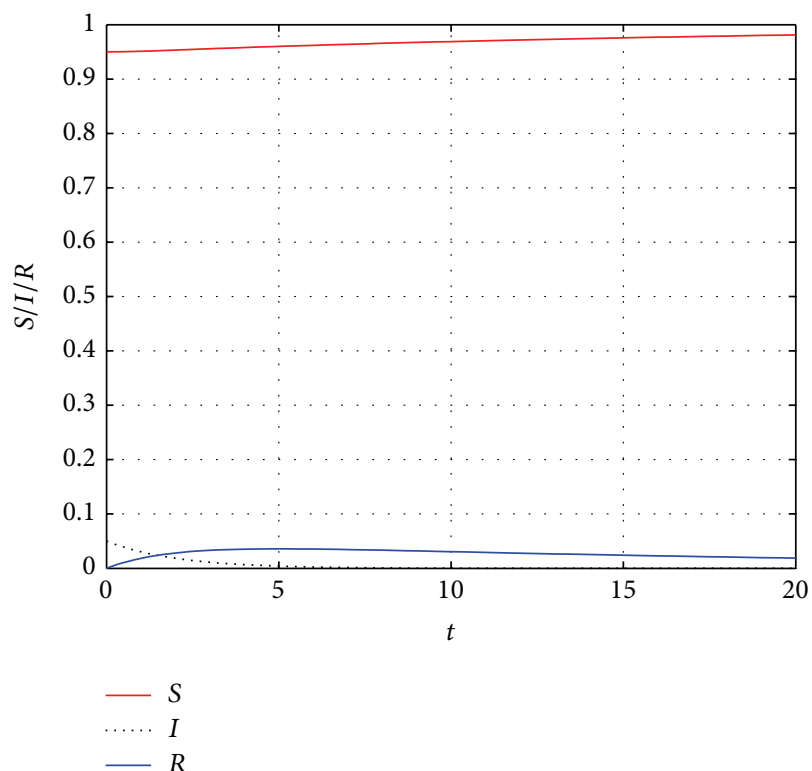

(c) Extended figure of (b)

(d) $\lambda=0.000001, \gamma=0.4, \beta=0.1, \varepsilon=0.1, \delta=0.05$, and $N=1400$

Figure 4: The dynamic variation of $S, I$, and $R$ (the figure on the right is the top 20 steps of the left).

when $t=1.502$ and reaches steady state on $42.38 \%$ with 593 infected passengers when $t=12.39$. With time step going on, the proportion of the susceptible persons $S(t)$ descends quickly while the proportion of the recovered persons $R(t)$ increases. And the trends of both curves of $S(t)$ and $R(t)$ finally reach steady state with $S^{*}=0.2119$. All the model parameters meet $1<-\langle k\rangle \rho \ln (1-\lambda) /(\gamma+\varepsilon)<1 / S^{*}$ and Theorem 2 is proved.

In order to verify Theorem 1 , we randomly assign model parameters as $\lambda=0.000001, \gamma=0.4, \beta=0.1, \varepsilon=0.1$, and $\delta=0.05$ to meet $-\langle k\rangle \rho \ln (1-\lambda) /(\gamma+\varepsilon)<1$. Simulation results are showed in Figures 4(c) and 4(d). The model finally reaches its stable point at $(S, I)=(1,0)$ and Theorem 1 is proved.

4.2. The Impact of Different Parameters on Panic. This section will use control variate method to analyze how model parameters influence the number of the infected people by separately changing infection rate, immunization rate, immune loss rate, and passenger number on Matlab simulation, based on the model parameters setting of Figures 4(a) and 4(b). The simulation results are shown in Figure 5. 


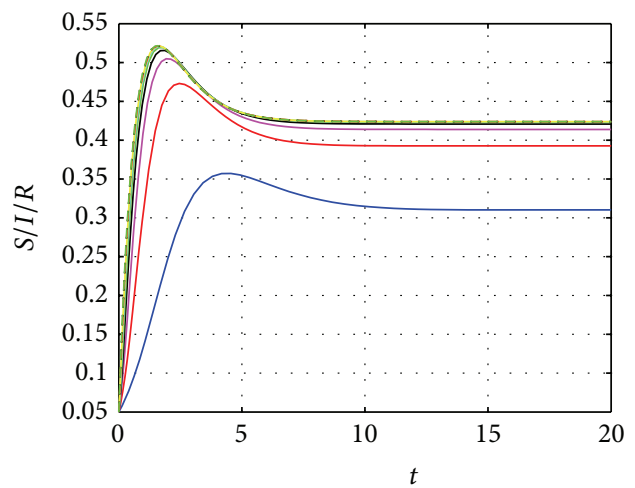

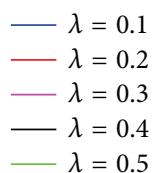

$$
\begin{aligned}
\lambda & =0.6 \\
\lambda & =0.7 \\
---\lambda & =0.8 \\
\cdots \cdots \lambda & =0.9 \\
---\lambda & =1
\end{aligned}
$$

(a) Changing infection rate

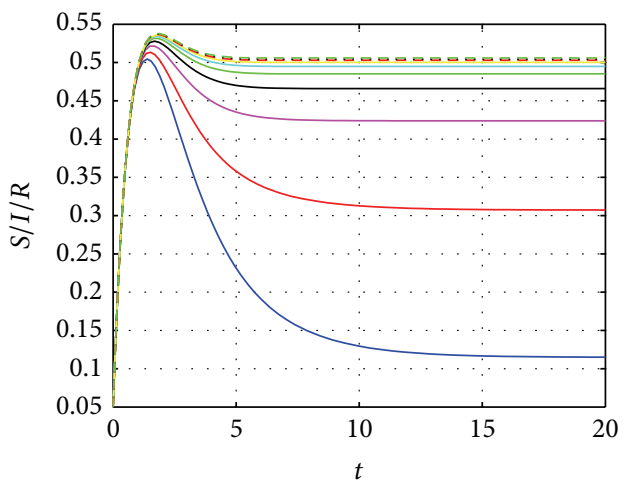

$$
\begin{aligned}
\beta & =0.25 \\
\beta & =0.3 \\
--\beta & =0.35 \\
\cdots \cdots \beta & =0.4 \\
---\beta & =0.9
\end{aligned}
$$

(c) Changing immune loss rate

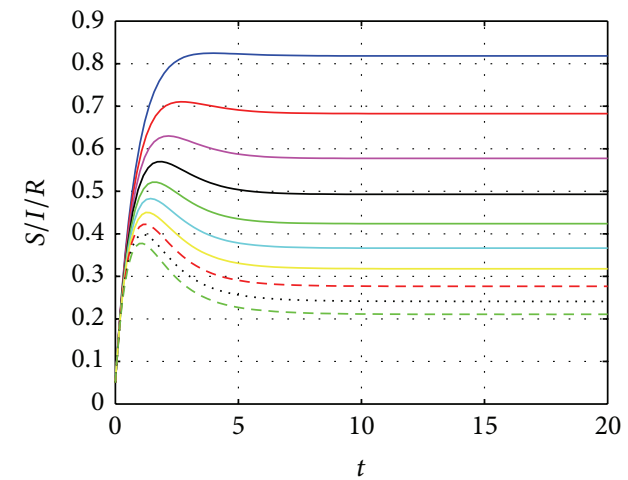

$$
\begin{array}{rlrl}
-\gamma & =0.1 & -\gamma=0.6 \\
\gamma=0.2 & \gamma=0.7 \\
\gamma=0.3 & --\gamma=0.8 \\
-\gamma=0.4 & \cdots \cdots \gamma=0.9 \\
-\gamma=0.5 & --\gamma=1
\end{array}
$$

(b) Changing immunization rate and spontaneous immunization rate

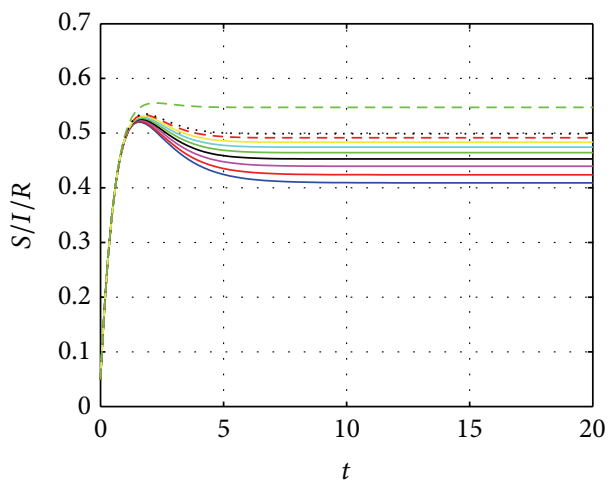

$$
\begin{aligned}
\delta & =0.01 \\
\delta & =0.05 \\
\delta & =0.1 \\
\delta & ---\delta=0.25 \\
\delta & =0.15 \\
-\ldots . \delta & =0.35 \\
\delta & =0.2
\end{aligned}
$$

(d) Changing spontaneous immune loss rate

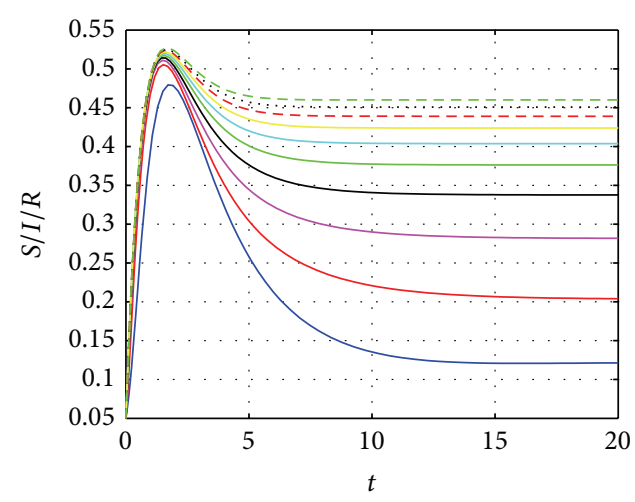

$$
\begin{aligned}
-N & =200 & -N & =1200 \\
N & =400 & -N & =1400 \\
N & =600 & ---N & =1600 \\
-N & =800 & \cdots N & =1800 \\
-N & =1000 & --N & =2000
\end{aligned}
$$

(e) Changing passenger number

FIGURE 5: Influence of the infected passengers number by different parameters. 


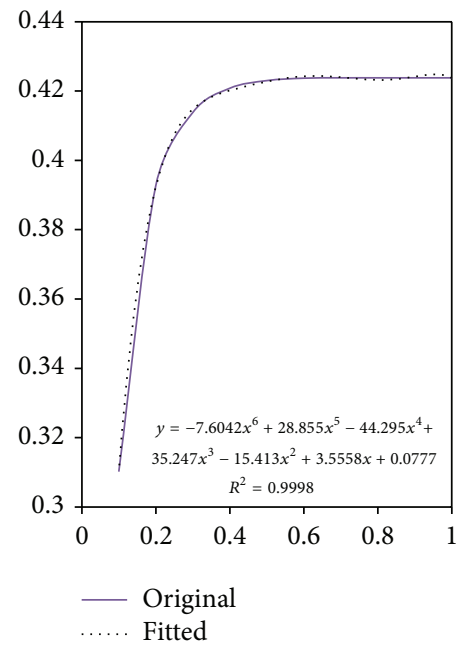

(a) Changing infection rate

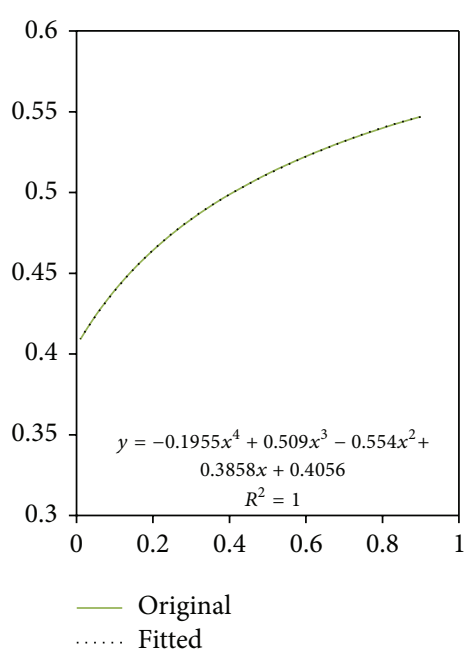

(d) Changing spontaneous immune loss rate

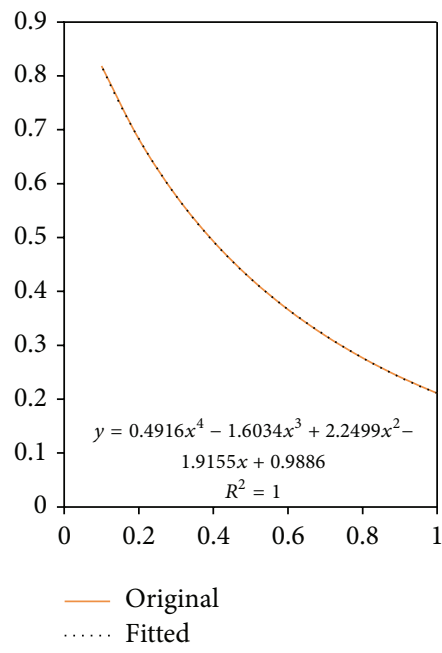

(b) Changing immunization rate and spontaneous immunization rate

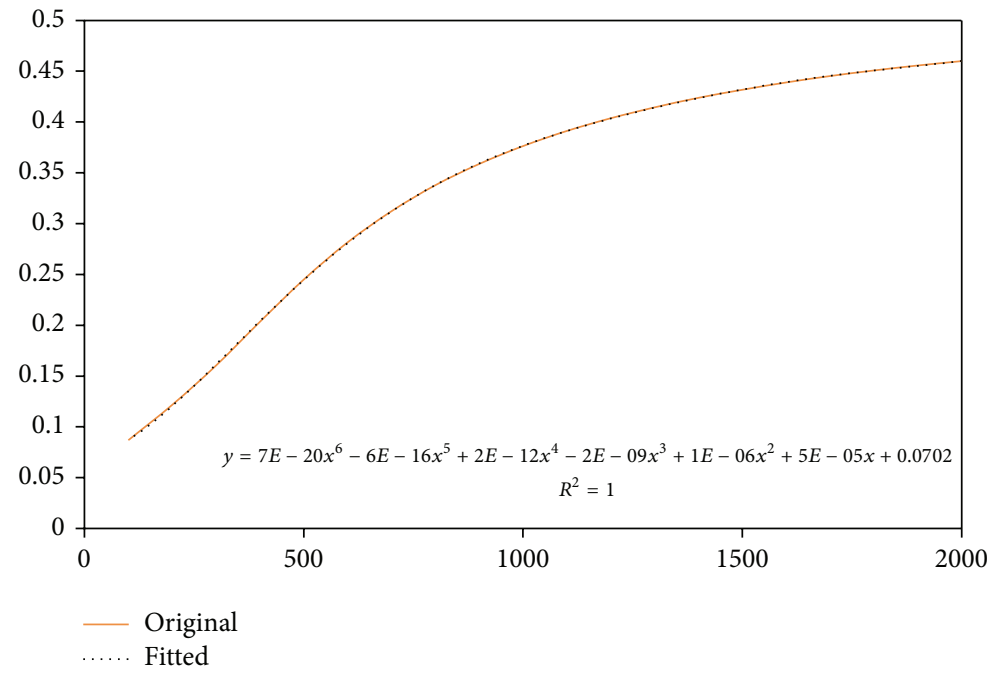

(e) Changing passenger number

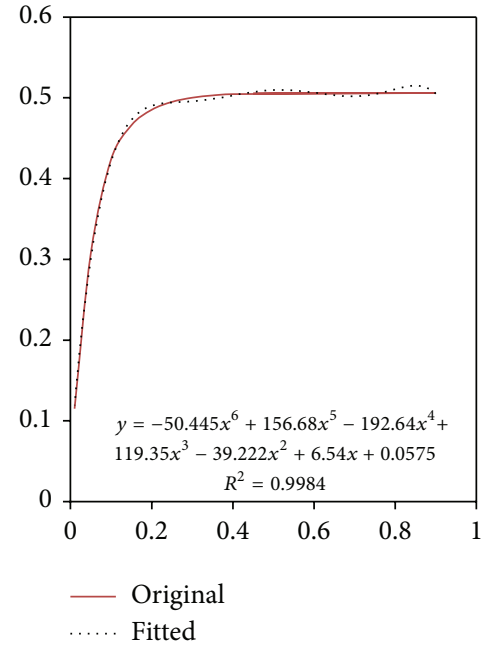

(c) Changing immune loss rate

FIGURE 6: Changing curves of the stable point by different parameters.

According to Figure 5, we can figure out that the changes of some parameters make the number of the infected passengers in steady state change greatly while the others change little. In order to reflect the relationships between parameters range and stable point, we draw the relationships in curves and fit them into equations, as shown in Figure 6.

In conclusion, according to Figures 5 and 6, we can figure that the infected proportion have a great influence on the final panic passengers number when the infected rate rises to 0.3 or the immunization rate goes to 0.2 , and after that the curve becomes gentle. Figure 6(d) shows that changing the spontaneous immune loss rate does not have so many effects on the final number of panic passengers; the proportion of infected people will be changed from 0.4 to 0.55 . However, if the immunization rates and spontaneous immunization rates are in a state of low value, passengers' panic spreads quickly 
TABLE 1: The impact of final infection rate and the amount of infected people by respectively changing multiple model parameters.

\begin{tabular}{|c|c|c|c|c|c|c|c|}
\hline No. & $\lambda$ & $\delta+\varepsilon$ & $\beta$ & $\delta$ & $N$ & Stable point & Infected amount \\
\hline 1 & 0.9 & 0.5 & 0.1 & 0.05 & 1400 & 0.4238 & 593 \\
\hline 2 & 0.8 & 0.5 & 0.1 & 0.05 & 1400 & 0.4238 & 593 \\
\hline 3 & 0.9 & 0.6 & 0.1 & 0.05 & 1400 & 0.3663 & 513 \\
\hline 4 & 0.8 & 0.6 & 0.1 & 0.05 & 1400 & 0.3663 & 513 \\
\hline 5 & 0.9 & 0.5 & 0.05 & 0.05 & 1400 & 0.3073 & 430 \\
\hline 6 & 0.8 & 0.5 & 0.05 & 0.05 & 1400 & 0.3095 & 433 \\
\hline 7 & 0.9 & 0.5 & 0.1 & 0.03 & 1400 & 0.4167 & 583 \\
\hline 8 & 0.8 & 0.5 & 0.1 & 0.03 & 1400 & 0.4167 & 583 \\
\hline 9 & 0.9 & 0.5 & 0.1 & 0.05 & 1000 & 0.3764 & 376 \\
\hline 10 & 0.8 & 0.5 & 0.1 & 0.05 & 1000 & 0.3762 & 376 \\
\hline 11 & 0.9 & 0.6 & 0.05 & 0.05 & 1400 & 0.2456 & 344 \\
\hline 12 & 0.8 & 0.6 & 0.05 & 0.05 & 1400 & 0.2454 & 344 \\
\hline 13 & 0.9 & 0.6 & 0.1 & 0.03 & 1400 & 0.3581 & 501 \\
\hline 14 & 0.8 & 0.6 & 0.1 & 0.03 & 1400 & 0.358 & 501 \\
\hline 15 & 0.9 & 0.6 & 0.1 & 0.05 & 1000 & 0.3154 & 315 \\
\hline 16 & 0.8 & 0.6 & 0.1 & 0.05 & 1000 & 0.3152 & 315 \\
\hline 17 & 0.9 & 0.5 & 0.05 & 0.05 & 1000 & 0.2418 & 242 \\
\hline 18 & 0.8 & 0.5 & 0.05 & 0.05 & 1000 & 0.2415 & 242 \\
\hline 19 & 0.9 & 0.5 & 0.1 & 0.03 & 1000 & 0.3647 & 365 \\
\hline 20 & 0.8 & 0.5 & 0.1 & 0.03 & 1000 & 0.3646 & 365 \\
\hline 21 & 0.9 & 0.6 & 0.05 & 0.03 & 1400 & 0.2211 & 310 \\
\hline 22 & 0.8 & 0.6 & 0.05 & 0.03 & 1400 & 0.2209 & 309 \\
\hline 23 & 0.9 & 0.6 & 0.1 & 0.03 & 1000 & 0.3017 & 302 \\
\hline 24 & 0.8 & 0.6 & 0.1 & 0.03 & 1000 & 0.3016 & 302 \\
\hline 25 & 0.9 & 0.5 & 0.05 & 0.03 & 1000 & 0.2085 & 209 \\
\hline 26 & 0.8 & 0.5 & 0.05 & 0.03 & 1000 & 0.2078 & 208 \\
\hline 27 & 0.9 & 0.6 & 0.05 & 0.03 & 1000 & 0.1512 & 151 \\
\hline 28 & 0.8 & 0.6 & 0.05 & 0.03 & 1000 & 0.1499 & 150 \\
\hline
\end{tabular}

comparison which shows that the immune loss rate is the most significant impact on the proportion of final infected proportion. In addition, comparing lines $1,2,4,12,22$, and 28 to each other, we conclude that the passenger density also has great influence on the infection proportion. However, the infection rate almost had no effects on the proportion of infected people between 0.3 and 0.9 .

The reduction of the degree of passengers' panic depends on the measures that the subway departments take. The safety management of the subway should be strengthened, national educational activities for subway emergencies should be carried out, and the passengers' number in the subway cars should be controlled strictly. Figure 7 is the propagation curve simulation with the parameters $\lambda=0.8, \gamma=0.6, \beta=$ $0.01, \varepsilon=0.01, \delta=0.05$, and $N=1000$.

The final numbers of the infected and the immune passengers have an obviously big difference by comparing Figure 7 with Figure 4(b). When the infected passengers' number in Figure 4(b) is 686, it reaches the stable state. The final infected passengers' proportion is $8.56 \%$ in Figure 7. They use different group numbers. While the former uses $N=1400$, the latter uses $N=1000$, so the number of infected passengers is about 86. Without the control strategies, the panic peak reaches $57 \%$ and the number of panic passengers is 798. By the use of the control strategies, the panic peak reaches $46.66 \%$ and the number of panic passengers is 467 , which suggests that the control strategies have greatly improved the panic peak and the final number of panic passengers.

\section{Conclusions}

This paper takes the spread characteristic of passengers panic under subway emergency into consideration and improves traditional SIRS model and parameters.

(1) The parameter, passengers' density $\rho$, which may change significantly in different time, is added in the model.

(2) In the subway car with strait and confined space, when the panic happens, it is probably not integrated panic, but the panic that spreads from the emergency car to another, which means that the distribution of panic passengers is not homogeneous. The infection rate and immune loss rate between passengers are determined by the surroundings. Therefore, it is more 


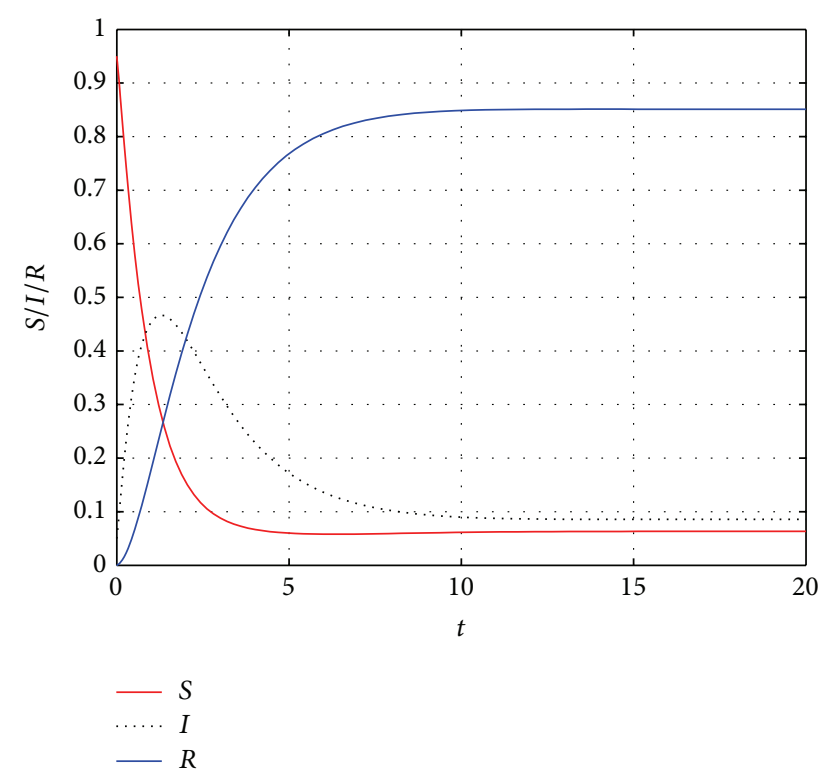

FIGURE 7: Result of integrated control strategies.

conformed to describe the infection rate and immune loss rate as

$$
\begin{aligned}
& G(t)=\left[1-(1-\lambda)^{\rho\langle k\rangle I(t)}\right], \\
& D(t)=\left[1-(1-\beta)^{\rho\langle k\rangle I(t)}\right] .
\end{aligned}
$$

(3) The influence of passenger psychological factors is needed to be considered, because the infected passengers possibly become spontaneously immune by their own psychological mentality while the immune passengers possibly become susceptible again by their own spontaneous immune loss characteristic. Therefore, the spontaneous immune parameters $\varepsilon$ and $\delta$ are added to the model to represent the spontaneous immune probability of the infected passengers and spontaneous immune loss probability of the immune passengers.

According to the three aspects above, the SIRS model of panic spread of passengers under subway emergency is built and is to be used to simulate the panic spread of the passengers, which reveals the rules of how group panic dynamic spread and verified the model stability. The trend of stable point of the infection rate is analyzed by changing different parameters and comes to a conclusion that immunization rate, spontaneous immune loss rate, and passengers' number had a great influence on the final infected passengers' number, rapidly reducing the effect of panic spread. Finally, this paper proposed integrated control strategies to strengthen the safety management of the subway, carry out national educational activities for subway emergencies, and strictly control the passengers' number in the subway car and made simulation to find that the passenger panic peak and the final infected passenger number were greatly improved. Currently, the model and conclusions are established under self-organizing subway emergencies. However, there are more factors that need to be considered, such as government or subway administration and some control strategies made by other organizations, which should be taken into account and it deserves more attention in the future research.

\section{Conflict of Interests}

The authors declare that there is no conflict of interests regarding the publication of this paper.

\section{Acknowledgments}

This research was supported by the NSFC Major Program (Grants nos. 71090404/71090400) and NSFC General Program (Grants nos. 71272045 and 71101107).

\section{References}

[1] S. L. Zhang, M. L. Liu, and E. M. Sun, "Comprehensive assessment on emergency evacuation capability of the subway station based on fuzzy network analysis," Applied Mechanics and Materials, vol. 357, pp. 2935-2939, 2013.

[2] T. Okumura, K. Suzuki, A. Fukuda et al., "The Tokyo subway sarin attack: disaster management, part 1: community emergency response," Academic Emergency Medicine, vol. 5, no. 6, pp. 613-617, 1998.

[3] L. Yan, W. Tong, Z. Wei, and W. Zongzhi, "Computer simulation of human emergency evacuation in subway station," in Proceedings of the 2nd International Conference on Risk Analysis and Crisis Response, pp. 513-519, 2009.

[4] Z. Zou and H. Guo, "Research on a fuzzy neural network based medical institutions selection in subway station emergency," Advanced Materials Research, vol. 361-363, pp. 1204-1210, 2012.

[5] P. Li, R. Lu, and F. Wang, "Study on supervision, alarm and emergency system for subway in big city," in Proceedings of the 2nd IEEE International Conference on Emergency Management and Management Sciences (ICEMMS '11), pp. 286-289, August 2011.

[6] L. Hu, L. Wu, K. Lu et al., "Optimization of emergency ventilation mode for a train on fire stopping beside platform of a metro station," Building Simulation, vol. 7, no. 2, pp. 137-146, 2014.

[7] C. S. Jiang, Y. Ling, C. Hu, Z. Yang, H. Ding, and W. K. Chow, "Numerical simulation of emergency evacuation of a subway station: a case study in Beijing," Architectural Science Review, vol. 52, no. 3, pp. 183-193, 2009.

[8] P. Yang, C. Li, and D. Chen, "Fire emergency evacuation simulation based on integrated fire-evacuation model with discrete design method," Advances in Engineering Software, vol. 65, pp. 101-111, 2013.

[9] J. Wan, J. Sui, and H. Yu, "Research on evacuation in the subway station in China based on the Combined Social Force Model," Physica A: Statistical Mechanics and Its Applications, vol. 394, pp. 33-46, 2014.

[10] W. Lei, A. Li, R. Gao et al., "Simulation of pedestrian crowds' evacuation in a huge transit terminal subway station," Physica A: Statistical Mechanics and Its Applications, vol. 391, no. 22, pp. 5355-5365, 2012.

[11] E. Quarantelli, “The behavior of panic participants," Sociology and Social Research, vol. 41, no. 3, pp. 187-194, 1957. 
[12] G. Le Bon, The Crowd: A Study of the Popular Mind, Macmillan, 1897.

[13] A. R. Mawson, "Is the concept of panic useful for study purposes," in Proceedings of the 2nd International Seminar on Behavior in Fire Emergencies, p. 29, National Bureau of Standards, Special Publication, 1978.

[14] B. E. Aguirre, "Emergency evacuations, panic, and social psychology," Psychiatry, vol. 68, no. 2, pp. 121-129, 2005.

[15] H. H. Kelley, J. C. Condry Jr., A. E. Dahlke, and A. H. Hill, "Collective behavior in a simulated panic situation," Journal of Experimental Social Psychology, vol. 1, no. 1, pp. 20-54, 1965.

[16] M. Ebihara, A. Ohtsuki, and H. Iwaki, "A model for simulating human behavior during emergency evacuation based on classificatory reasoning and certainty value handling," ComputerAided Civil and Infrastructure Engineering, vol. 7, no. 1, pp. 6371, 1992.

[17] C. Saloma, G. J. Perez, G. Tapang, M. Lim, and C. PalmesSaloma, "Self-organized queuing and scale-free behavior in real escape panic," Proceedings of the National Academy of Sciences of the United States of America, vol. 100, no. 21, pp. 11947-11952, 2003.

[18] D. J. Low, "Following the crowd," Nature, vol. 407, no. 6803, pp. 465-466, 2000.

[19] D. Helbing, I. Farkas, and T. Vicsek, "Simulating dynamical features of escape panic," Nature, vol. 407, no. 6803, pp. 487490, 2000.

[20] M. Kermack and A. Mckendrick, "Contributions to the mathematical theory of epidemics: part II," Proceedings of the Royal Society A, vol. 138, pp. 55-83, 1932.

[21] M. Kermack and A. Mckendrick, "Contributions to the mathematical theory of epidemics: part I," Proceedings of the Royal Society A, vol. 115, pp. 700-721, 1927.

[22] C. H. Li, C. C. Tsai, and S. Y. Yang, "Analysis of epidemic spreading of an SIRS model in complex heterogeneous networks," Communications in Nonlinear Science and Numerical Simulation, vol. 19, no. 4, pp. 1042-1054, 2014.

[23] B. Dybiec, "SIR model of epidemic spread with accumulated exposure," European Physical Journal B, vol. 67, no. 3, pp. 377383, 2009.

[24] L. Zhao, Q. Wang, J. Cheng, Y. Chen, J. Wang, and W. Huang, "Rumor spreading model with consideration of forgetting mechanism: a case of online blogging Live Journal," Physica A: Statistical Mechanics and Its Applications, vol. 390, no. 13, pp. 2619-2625, 2011.

[25] L. Zhao, J. Wang, Y. Chen, Q. Wang, J. Cheng, and H. Cui, "SIHR rumor spreading model in social networks," Physica A: Statistical Mechanics and Its Applications, vol. 391, no. 7, pp. 2444-2453, 2012.

[26] L. J. Zhao, H. X. Cui, X. Y. Qiu, and X. L. Wang, "SIR rumor spreading model in the new media age," Physica A: Statistical Mechanics and Its Applications, vol. 392, no. 4, pp. 995-1003, 2013.

[27] F. R. Durand, M. L. F. Abbade, E. Moschim et al., "SIR optimization in wavelength-hopping time spreading optical code routed networks," Optik, vol. 124, no. 18, pp. 3208-3214, 2013.

[28] Y. Maki and H. Hirose, "Infectious disease spread analysis using stochastic differential equations for SIR model," in Proceedings of the 4th IEEE International Conference on Intelligent Systems Modelling \& Simulation (ISMS '13), pp. 152-156, 2013.
[29] D. F. Bernardes, M. Latapy, and F. Tarissan, "Relevance of SIR model for real-world spreading phenomena: experiments on a large-scale $\mathrm{p} 2 \mathrm{p}$ system," in Proceedings of the International Conference on Advances in Social Networks Analysis and Mining (ASONAM '12), pp. 327-334, IEEE Computer Society, 2012.

[30] S. Bargmann and P. M. Jordan, "A second-sound based, hyperbolic SIR model for high-diffusivity spread," Physics Letters A: General, Atomic and Solid State Physics, vol. 375, no. 5, pp. 898907, 2011.

[31] S.-K. Zhang, S.-R. Gong, and Z.-M. Cui, "Study on spreading of virus infection with SIRS characteristic in wireless sensor networks," in Proceedings of the WRI International Conference on Communications and Mobile Computing (CMC '09), vol. 3, pp. 512-517, January 2009.

[32] H. Fukś, A. T. Lawniczak, and R. Duchesne, "Effects of population mixing on the spread of SIR epidemics," European Physical Journal B, vol. 50, no. 1-2, pp. 209-214, 2006.

[33] H. W. Hethcote, "Mathematics of infectious diseases," SIAM Review, vol. 42, no. 4, pp. 599-653, 2000.

[34] R. Pastor-Satorras and A. Vespignani, "Epidemic spreading in scale-free networks," Physical Review Letters, vol. 86, no. 14, pp. 3200-3203, 2001.

[35] A. Ramani, A. S. Carstea, R. Willox, and B. Grammaticos, "Oscillating epidemics: a discrete-time model," Physica A: Statistical Mechanics and Its Applications, vol. 333, no. 1-4, pp. 278-292, 2004.

[36] C. Piccardi and R. Casagrandi, "Inefficient epidemic spreading in scale-free networks," Physical Review E: Statistical, Nonlinear, and Soft Matter Physics, vol. 77, no. 2, Article ID 026113, 2008.

[37] R. Pastor-Satorras and A. Vespignani, "Epidemic dynamics and endemic states in complex networks," Physical Review E: Statistical, Nonlinear, and Soft Matter Physics, vol. 63, no. 6, Article ID 066117, 2001.

[38] Y. Moreno, J. B. Gómez, and A. F. Pacheco, "Epidemic incidence in correlated complex networks," Physical Review E: Statistical, Nonlinear, and Soft Matter Physics, vol. 68, no. 3, Article ID 035103, 2003.

[39] Y. Moreno, M. Nekovee, and A. F. Pacheco, "Dynamics of rumor spreading in complex networks," Physical Review E: Statistical, Nonlinear, and Soft Matter Physics, vol. 69, no. 6, Article ID 066130, 2004.

[40] C.-H. Li, C.-C. Tsai, and S.-Y. Yang, "Analysis of the permanence of an SIR epidemic model with logistic process and distributed time delay," Communications in Nonlinear Science and Numerical Simulation, vol. 17, no. 9, pp. 3696-3707, 2012.

[41] M. Yuanyuan, Z. Xintian, and L. Linguan, "SusceptibleInfected-Removed (SIR) model of crisis spreading in the correlated network of listed companies and their main stockholders.," Journal of Management Sciences in China, vol. 16, no. 7, pp. 80-94, 2013.

[42] M. Sekiguchi and E. Ishiwata, "Global dynamics of a discretized SIRS epidemic model with time delay," Journal of Mathematical Analysis and Applications, vol. 371, no. 1, pp. 195-202, 2010.

[43] J. M. Tchuenche, A. Nwagwo, and R. Levins, "Global behaviour of an SIR epidemic model with time delay," Mathematical Methods in the Applied Sciences, vol. 30, no. 6, pp. 733-749, 2007.

[44] J. M. Tchuenche and C. Chiyaka, "Global dynamics of a time delayed SIR model with varying population size," Dynamical Systems, vol. 27, no. 2, pp. 145-160, 2012.

[45] A. D'Onofrio, "On pulse vaccination strategy in the SIR epidemic model with vertical transmission," Applied Mathematics Letters, vol. 18, no. 7, pp. 729-732, 2005. 
[46] S. Gao, D. Xie, and L. Chen, "Pulse vaccination strategy in a delayed SIR epidemic model with vertical transmission," Discrete and Continuous Dynamical Systems B, vol. 7, no. 1, pp. 77-86, 2007.

[47] Z. Lu, X. Chi, and L. Chen, "The effect of constant and pulse vaccination on SIR epidemic model with horizontal and vertical transmission," Mathematical and Computer Modelling, vol. 36, no. 9-10, pp. 1039-1057, 2002.

[48] J. Li and N. Cui, "Dynamic behavior for an SIRS model with nonlinear incidence rate and treatment," The Scientific World Journal, vol. 2013, Article ID 209256, 5 pages, 2013.

[49] Y. Lin and D. Jiang, "Dynamics of a multigroup SIR epidemic model with nonlinear incidence and stochastic perturbation," Abstract and Applied Analysis, vol. 2013, Article ID 917389, 12 pages, 2013.

[50] Z. Hu, W. Ma, and S. Ruan, "Analysis of SIR epidemic models with nonlinear incidence rate and treatment," Mathematical Biosciences, vol. 238, no. 1, pp. 12-20, 2012.

[51] J. Li, N. Cui, and H. Sun, "Dynamic behavior for an SIRS model with nonlinear incidence rate," Advanced Materials Research, vol. 479-481, pp. 1495-1498, 2012. 


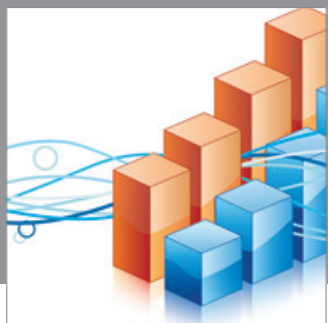

Advances in

Operations Research

mansans

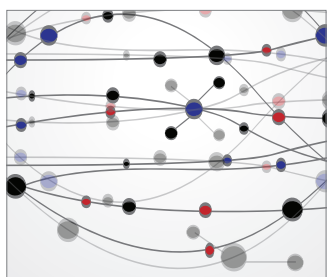

The Scientific World Journal
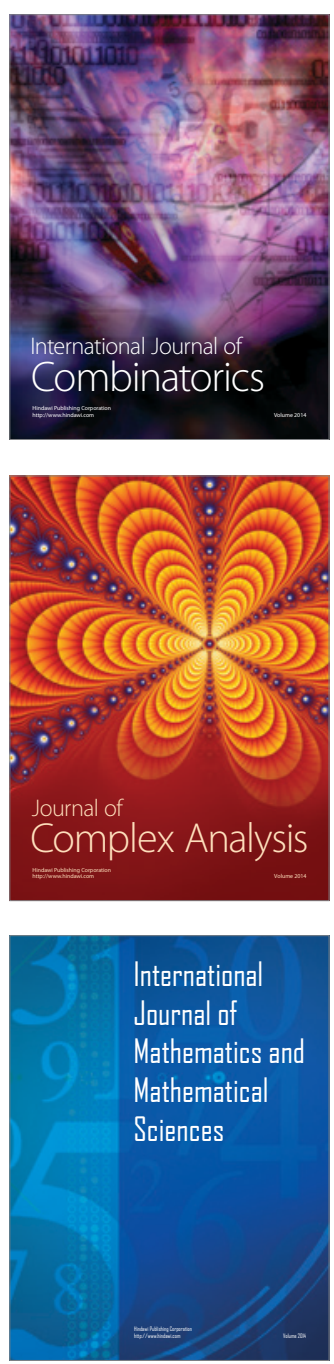
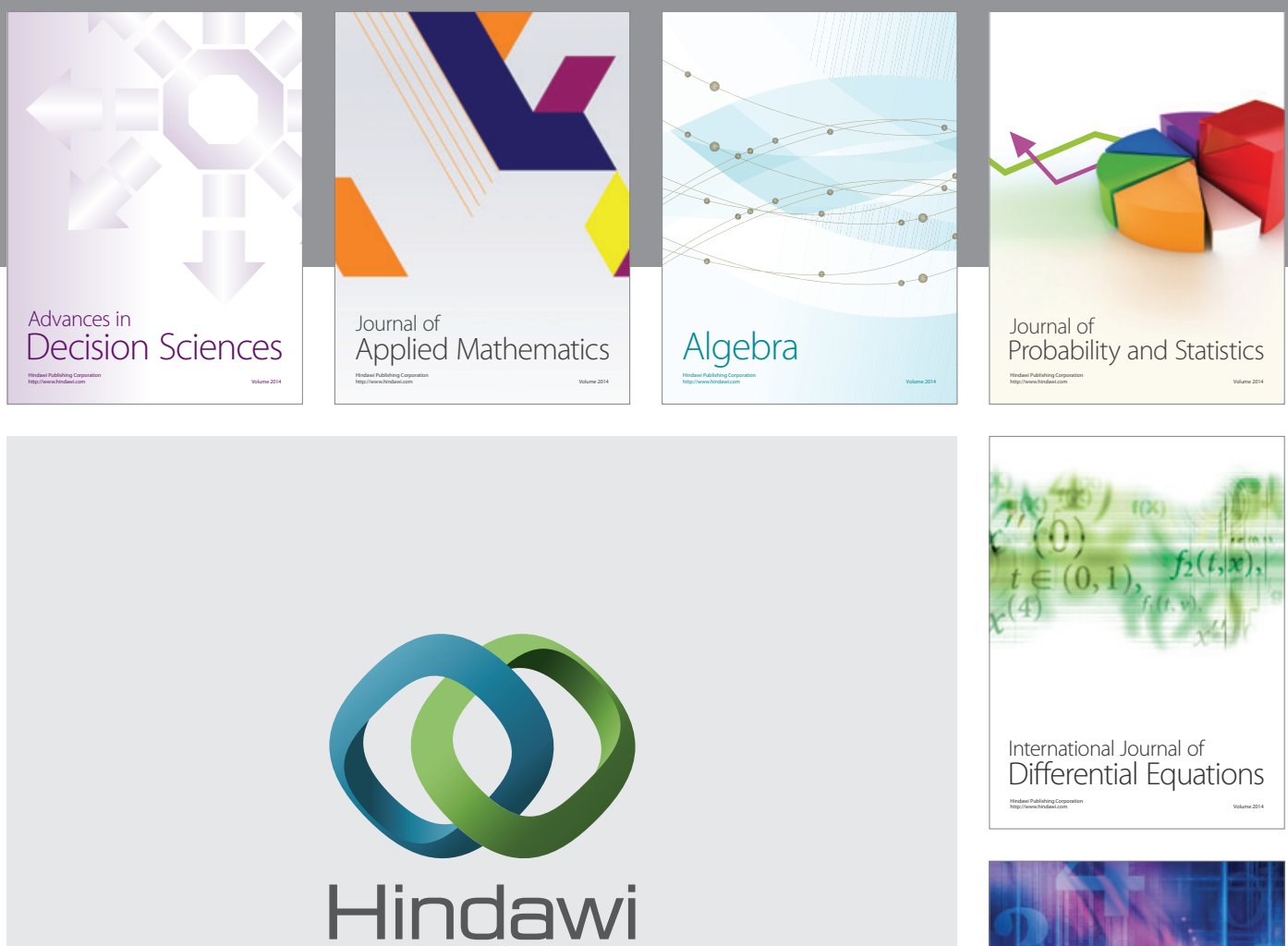

Submit your manuscripts at http://www.hindawi.com
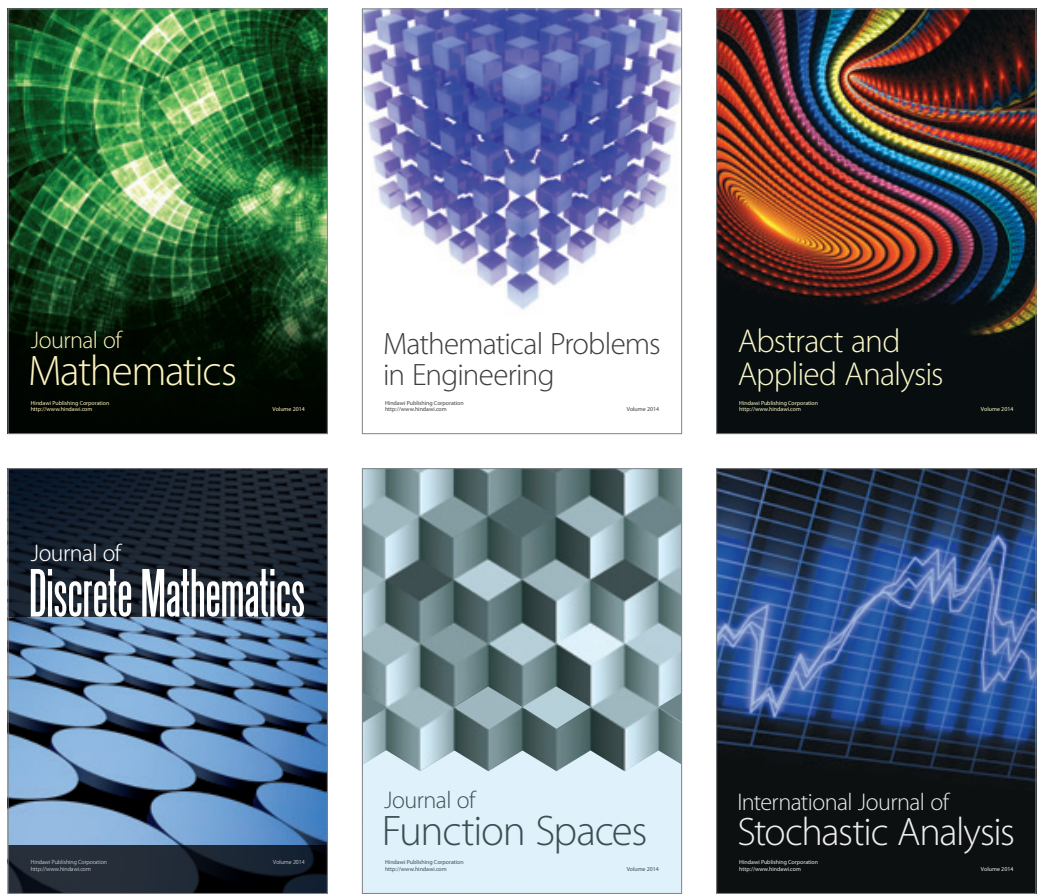

Journal of

Function Spaces

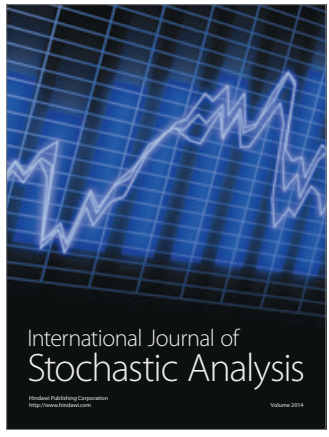

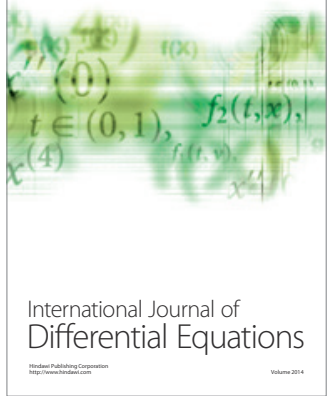
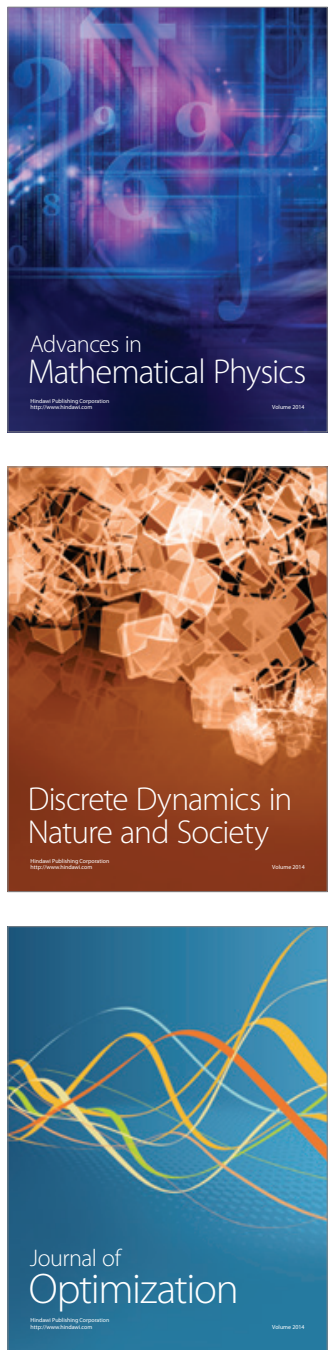